\title{
Assembly and Packaging of a Wireless, Chronically-Implantable Neural Prosthetic Device
}

K. G. Shah, T. DeLima, W. Benett, S. Felix, H. Sheth, V. Tolosa, A. Tooker, S. Pannu

August 8, 2012

International Microelectronics and Packaging Society San Diego, United States September 10, 2012 through September 13, 2012 
This document was prepared as an account of work sponsored by an agency of the United States government. Neither the United States government nor Lawrence Livermore National Security, LLC, nor any of their employees makes any warranty, expressed or implied, or assumes any legal liability or responsibility for the accuracy, completeness, or usefulness of any information, apparatus, product, or process disclosed, or represents that its use would not infringe privately owned rights. Reference herein to any specific commercial product, process, or service by trade name, trademark, manufacturer, or otherwise does not necessarily constitute or imply its endorsement, recommendation, or favoring by the United States government or Lawrence Livermore National Security, LLC. The views and opinions of authors expressed herein do not necessarily state or reflect those of the United States government or Lawrence Livermore National Security, LLC, and shall not be used for advertising or product endorsement purposes. 


\title{
Assembly and Packaging of a Wireless, Chronically-Implantable Neural Prosthetic Device
}

\author{
Kedar G. Shah, A. Tooker, V. Tolosa, T. DeLima, W. Benett, S. Felix, H. Sheth, S. Pannu \\ Lawrence Livermore National Laboratory \\ Center for Micro- and Nano-Technology \\ 7000 East Ave, Livermore, CA 94550 \\ Email: kshah@llnl.gov, Phone: +1 925.422.7296
}

\begin{abstract}
:
We present a chronically implantable, 121-channel, wireless neural prosthetic device assembled using a combination of novel and standard microelectronics packaging techniques. The exposed components are made of long-term biocompatible materials and are assembled into a three-dimensional package to minimize implant size. This device will be implanted into the spinal cord of an animal and is capable of electrically stimulating neural tissue. The objective of this work is to develop a clinical treatment for patients who lack micturition or bladder control due to paralysis or neurological-impairment.
\end{abstract}

Keywords: neural interface, neural prosthesis, bio-medical packaging, biocompatible packaging

\section{Introduction and Motivation:}

There is a growing need for bio-compatible devices that electrically stimulate or record signals from neural tissue. Such neural prosthetic devices enable treatment of chronic neurological disorders such as deafness, blindness, spinal cord injury, and brainrelated diseases. Most current research focuses on the neural interface, with percutaneous devices that are tethered to the patient. Though such technologies are critical to understanding neurological disorders, they do not offer a viable path to clinical treatment.

This work presents the development of a fully wireless, chronically-implantable neural prosthesis. The device has a high-density array of electrodes for stimulation in the spinal cord, to restore function to neurologically-impaired patients.

\section{Device Design and Assembly:}

The neural prosthetic consists of three components:

1. High-density microelectrode array

2. Hermetically sealed electronics package

3. Wireless data and power telemetry system.

These components are assembled into a chronically implantable device using a combination of custom and standard processes.

The microelectrode array consists of 121 stimulating electrodes. The array is built by creating a sandwich structure of alternating layers of polyimide (insulator and structural layer) and metal (electrical conductor).
Multiple metal layers are stacked to minimize the width of the array in order to reduce tissue damage during and after implantation. The microelectrode array has iridium oxide electrodes that electrically interface with the neural tissue. The image below details the process steps to microfabricate a polyimide-based, three-layer metal structure. The resulting device has passed ISO 10993 biocompatibility testing protocol, which makes is suitable for long-term implantation.

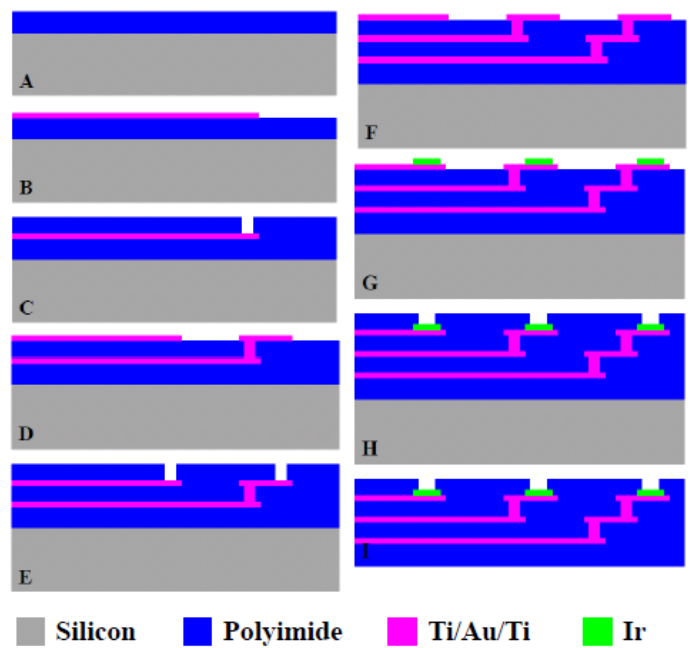

Figure 1: Cross-sectional view of the general fabrication process for three-layer trace metal, polyimide microelectrode arrays. (Images are not drawn to scale.) 

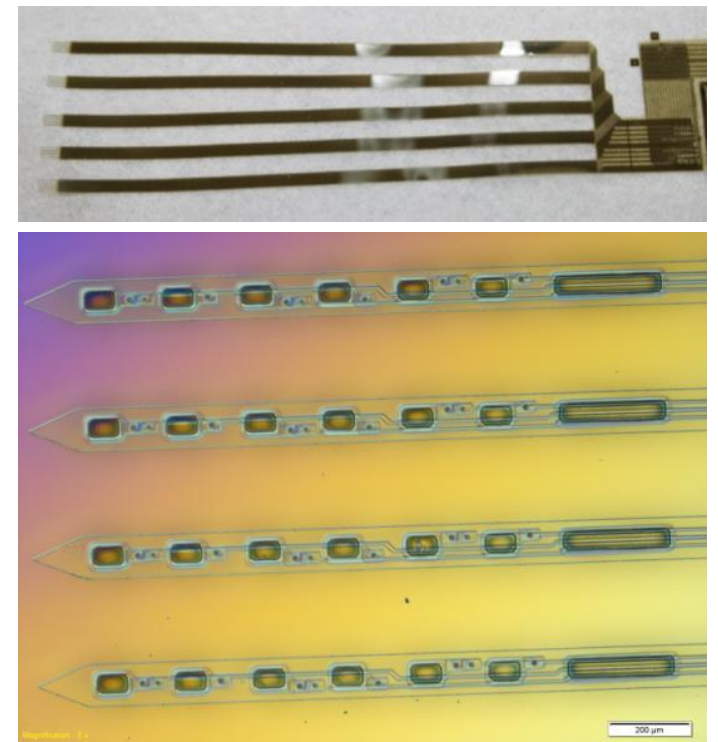

Figure 2: The full microelectrode array, with 5 probes (top image). All of the probes join at a single connector (right). An enlargement of one of the probe tips (bottom image) shows 28 recording/stimulating electrodes.

A variety of electronic components are used to convert the wireless data and power signals into the 121 individual electrical impulses that are delivered to the neural tissue:

1. A stimulator chip uses incoming data signals to generate independent electrical impulses that are delivered to the tissue through the microelectrode array.

2. An inductively-coupled power telemetry chip powers the stimulator chip

3. Associated surface mount circuitry (both active and passive), such as an oscillator (for the chip clock), resistors, capacitors, diodes, and an inverter.

These electronic components need to be sealed and separated from the neural tissue so that the nonbiocompatible components do not elicit a cytotoxic response in the tissue. Additionally, the package needs to be hermetically sealed in order to prevent corrosion or shorting of electrical components in the harsh biological environment of the body.

The electronic components are assembled using several packaging/assembly methods to minimize the total volume of the device. These components are assembled inside a hermetically-sealed package. Electrical signals are transmitted through a highdensity feedthrough array to the microelectrode array. The ceramic feedthrough array contains hermetic platinum vias and is metalized and patterned on both sides with a biocompatible multi-layer metal stack. The stimulator chip is assembled directly on the ceramic substrate with flip-chip bonding of gold stud bumps. The remainder of the electronics components (power telemetry chip and other circuitry) are assembled onto an interconnect board. High-aspectratio wire-bonds serve as interconnects between the power telemetry chip, the interconnect board, and the stimulator chip. The ceramic substrate is brazed to a biocompatible metal ring. Once all of the electronics components are assembled onto the ceramic substrate, the microelectronics package is hermetically-sealed by laser welding a metal cap to the ring. Finally, the microelectrode array and inductive coils are electrically attached to the ceramic substrate.

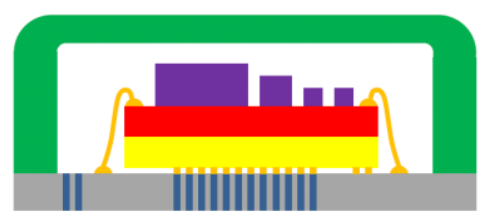

Figure 3: Schematic cross-section of the electronic package showing the general package architecture.

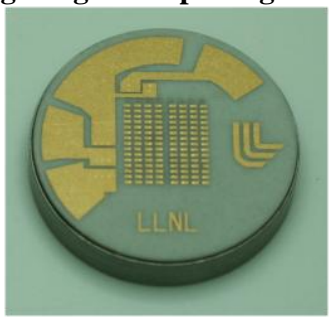

Figure 4: The metalized ceramic feedthrough array brazed to a biocompatible metal can. The electronic components are assembled inside this package.

This device does not contain a battery to create electrical stimulation pulses. Instead, inductively coupled coils are attached to the metal pads on the exterior of the package. These coils receive wireless data and power signals from an external wireless stimulator. The coils are wound with gold wire coated with parylene and polyimide.

\section{Discussion:}

LLNL has developed the packaging and assembly modules that are required to build the neural prosthetic. In the upcoming months, we will perform in-vitro testing of the neural prosthesis. Upon verifying functionality, prototypes will be built and implanted into an animal model to perform a chronic study for micturition control.

\section{Acknowledgements:}

Professor Wentai Liu's group at the University of California, Santa Cruz, for the microelectronics.

This work performed under the auspices of the U.S. Department of Energy by Lawrence Livermore National Laboratory under contract DE-AC5207NA27344. 